Journal of Computer Science 8 (8): 1244-1252, 2012

ISSN 1549-3636

(C) 2012 Science Publications

\title{
Context Aware Middleware Model for Handling Vehicular Safety Issues
}

\author{
Arunkumar Thangavelu and R.A.K. Saravanaguru \\ School of Computing Science and Engineering, VIT University, 632014, India
}

\begin{abstract}
Problem statement: This study proposed a mathematical model for the existing framework InVANET; context prediction and simulation for vehicle safety. Approach: Markov process was used to model context and predict the states with the help of transition probability matrix. The context parameters were collected from CarSim simulator and inferred using JENA and SPARQL. Results: The longitudinal speed and lateral offset are mainly considered and analyzed for a distracted driver situation. Conclusion: The importance of middleware and modeling is discussed and simulation shows the performance of the proposed model for handling vehicle safety issues.
\end{abstract}

Key words: In VANET, context aware middleware, markov model, vehicle safety, context prediction, Markov process, lateral offset, probability matrix, distracted driver

\section{INTRODUCTION}

World Health Organization global status report on road safety (WHO, 2009) treated road safety issues as Global Health Problem; also shows every minute a person die in road accident worldwide and the reason for the accidents are due to different traffic situations and road users.

Most National and International level transportation projects efforts have seen a greater focus on vehicle safety. There are many initiatives under Intelligent Transportation Systems (ITS) like PROMETHEUS (Williams, 1988), CarTALK (Reichardt et al., 2002), Inter-Vehicle Hazard Warning IVHW (Benoit, 2002), FleetNet (Andreas et al., 2004), WILLWARN (Gerhard, 2004), PATH project (Shladover, 2007), VETRAC (Arunkumar and Sivanandam, 2007) and NOW (Festag et al., 2008).

Transportation Literature Search by WorldCat, USDOT and NTL during the year 2010 stated that the potential for increased injuries or crash severity due to large passenger vehicles sharing the road with smaller passenger vehicles. So, predicting the impact of criticality based on vehicle type, current activity and alerts the driver or road users accordingly will prevent from crashes and also minimizes the damages caused by collisions in worst case situations (Sadayuki, 2011). Intelligent model for traffic safety applications discussed the decision making issues in different traffic scenarios. (Nagappan and Chellappan, 2012).

We categorize the crash predictions on highway into two types: The first type is by collecting the historical crash data with the related context information such as time, location, weather condition, drunken driving and others. This method of prediction is not suitable for time critical situations and only well in advance states with absolute time such as Calendar or time duration of an activity.

The second type is predicting the criticality of the situation by observing the current state of driver through different soft computing techniques like logic design, fuzzy system, neural network and artificial intelligence.

InVANET (Saravanan et al., 2009) focuses on the second type of prediction that incorporates intelligence into vehicular system, which can be employed in various safety scenarios such as minimizing vehicular accidents, managing vehicular traffic intensity, information for traffic cops on hit and run, avoiding vehicle theft and locating vehicles on drive way.

This study highly focuses on modeling context prediction and sharing over intelligent middleware system InVANET, where predicting the context information becomes a challenging task between high speed vehicles and also sharing context information plays major role with different traffic scenarios.

This study covers the following topics: an overview of existing InVANET architecture and thorough review of related work. The context inference and sharing mechanism are discussed. A simulated experimental test-bed using CarSim and JENA to evaluate the middleware and also discussed the performance analysis

InVANET and related work: InVANET middleware (Saravanan et al., 2009) architecture Fig. 1a adopts object based component modeling where each module inter operates with each other in decision making and policy generation. 
(Wheel movement, steering control, brake, gear info, indicator info)

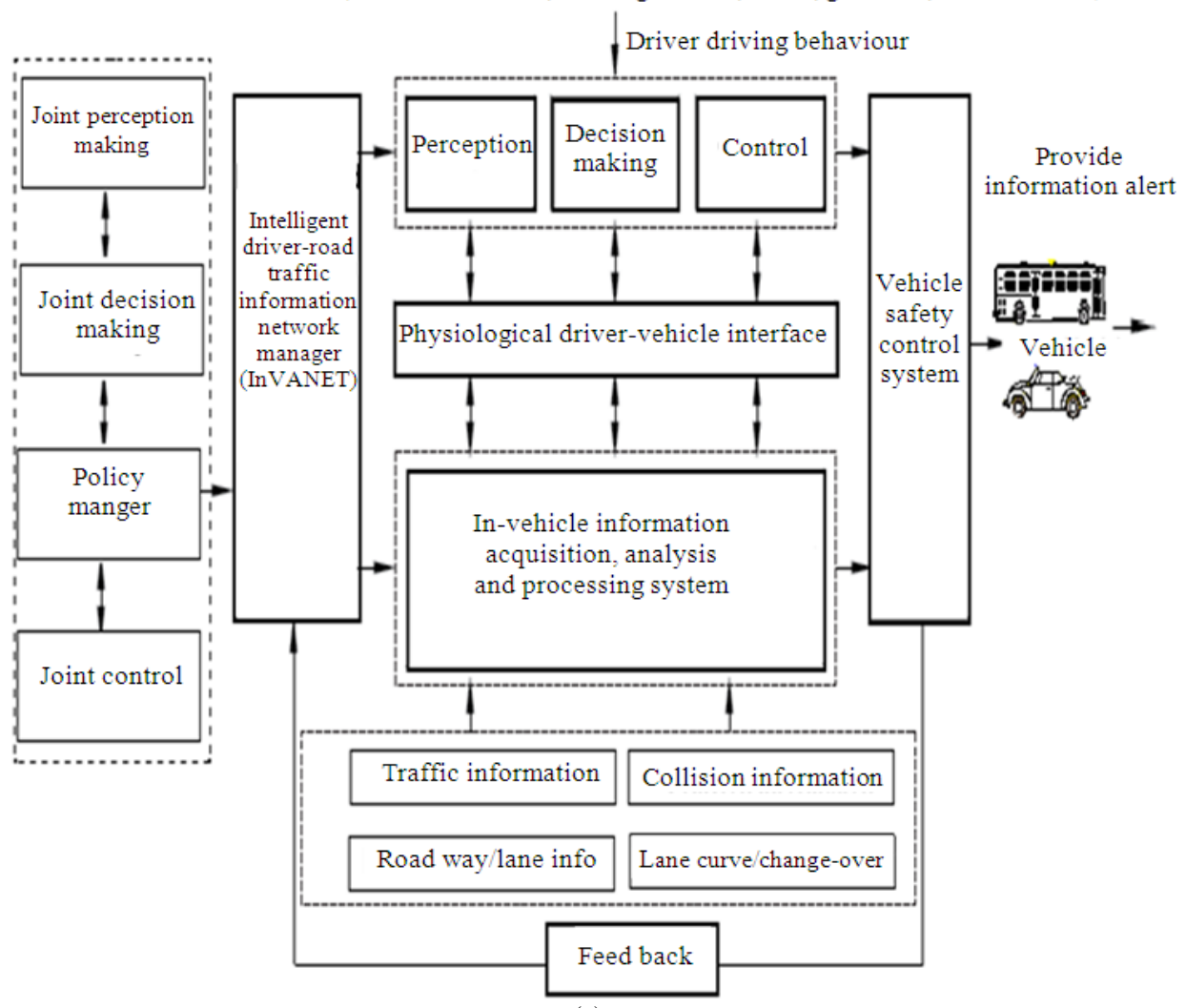

(a)

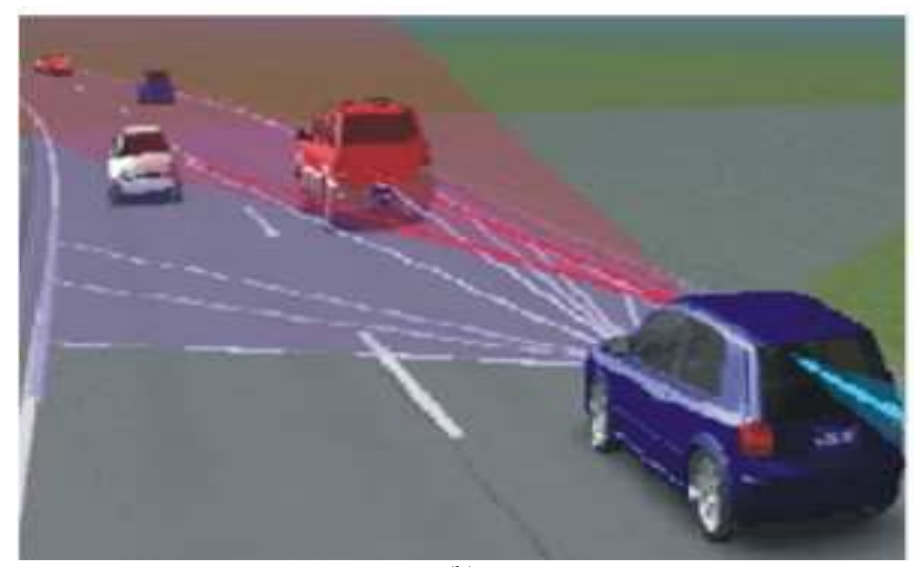

(b)

Fig. 1: (a) Architecture InVANET (Intelligence Vehicular Ad-Hoc Network) (b) Curvy road with lane edge information

The primary modules of InVANET focus on:

- Gathering driver's driving physiological scenario, gathering roadway lane information

- Enabling policy based decision making based on contextual information and scenario
- Gather and update vehicular context for variable roadway scenario in XML format being consistently update maintained from ontology

In this research study, all the above mentioned parameters considered as Context and derive information from the context for modeling InVANET architecture. 


\section{J. Computer Sci., 8 (8): 1244-1252, 2012}

NHTSA Survey (Wassim et al., 2007) conducts vehicle safety research in crash avoidance; discusses 37 pre crash scenarios which promote researchers to investigate and develop crash avoidance systems.

The context change can be notified with the help of event based middleware, publish/subscribe middleware and adaptive middleware in dynamic changing environment but difficult is considering sensitive situational parameters.

CORTEX (Gura et al., 2001) middleware project was implemented for providing proactive services in a distributed traffic telemeatics application with the help of JMF and RTP. Predictability of the communication and cooperation between entities was mentioned as future work.

ScudWare (Zhaohui et al., 2007), CARS (Shankar et al., 2008), CARM (Shengpu and Liu, 2011) were proposed as middleware architectures for the following vehicular applications; run time provisioning for authentication, telematics applications and to perform dynamic configuration of components respectively; As the complexity increases to analyze critical states the majority of solutions only provided for telematics in vehicles and less intent to vehicle safety.

Figure 1b shows, when the vehicle turns on the curvy road the necessary context needed to be observed like lane edge and surrounding object information.

Markov model for context analysis: Though publish/subscribe and event based middleware suffice in providing services between vehicles, predicting the situation without any cessation, analyze about the criticality is more intricate to process. The situation becomes critical through the unforeseen events like child running across the road, aggressive driving, diverts the attention due to talking on mobile phone, road conditions and vehicle faults. Achieving this goal requires prediction of context with the help of recording the observations at a specific time interval to understand about the situation.

Finite State Markov model is a simple and effective approach for communication channel description (Babich and Lombardi, 2000). Six states are considered in markov process for context-aware data communication to model context prediction over InVANET middleware.

The basic idea of using markov process is to solve the high mobility issues in vehicular models and the markov property is appropriate to deploy such applications.

We consider the Markov process, in which the state at time $t+1$ depends on the states at time $t$. Thus the future state of the process depends only the current state and not by past states. i.e., given the past states $\mathrm{X}_{0}, \mathrm{X}_{1}, \ldots . \mathrm{X}_{\mathrm{n}-1}$ and present state $\mathrm{X}_{\mathrm{n}}$ the future state $\mathrm{X}_{\mathrm{n}+1}$ is independent of past states and depends only on present state. The transition from the current state ' $i$ ' to next state ' $\mathrm{j}$ ' is based on the probability that process. The sequence of States $\left\{X_{0} \ldots X_{n}\right\}$ forms a $(n+1)$-state markov chain with its one-step transition probability matrix $P$ given by $P=\left\{P_{i j}\right\}>=0$, such that $i, j>=0$ :

$$
P\left\{\begin{array}{c}
X_{n+1}=j \mid X_{n}=i, X_{n-1}= \\
i_{n-1}, \ldots X_{1}=i 1, X_{0}=i_{0}
\end{array}\right\}=P_{i j}
$$

State diagram: The Situation $(\mathrm{S})$ represented as 'state' in markov process is defined as composition of Object (O), Time (T) and Context (C) from the InVANET components. Object is used as the functional element to represent the vehicle dynamics based on longitudinal and lateral control.

- $\quad$ Situation $S=\{\mathrm{O}, \mathrm{T}, \mathrm{C}\}$ where $\mathrm{O}, \mathrm{T}, \mathrm{C}$ are abstract form of object, time and context

- $\quad$ Object $\mathrm{O}=\left\{\mathrm{O} 1, \mathrm{O} 2, \mathrm{O} 3 \ldots \mathrm{O}_{\mathrm{n}}\right\}$ set of objects involved in sensing and sharing of context information

- $\quad$ Time $\mathrm{T}=\left\{\mathrm{T} 1, \mathrm{~T} 2, \mathrm{~T} 3 \ldots \mathrm{T}_{\mathrm{n}}\right\}$ time duration values at instance ' $n$ ' (used in simulation for sampling)

- Context $\left.\mathrm{C}=\mathrm{C} 1, \mathrm{C} 2, \mathrm{C} 3 \ldots \mathrm{C}_{\mathrm{n}}\right\}$ set of context information used to describe the current situation

The State space ' $S$ ' and Transition Probability Matrix 'TPM' for Markov model represented as follows:

State space $S=\left\{S_{1}, S_{2}, S_{3}, S_{4}, S_{5}, S_{6}\right\}$

\section{State of vehicle:}

S1: Initial state (Stopped State: starting vehicle, speed $=0)$

S2: Casual state (No Alert State: No Vehicle ahead)

S3 Alert state (Beep or Alert: Vehicle/obstacle ahead, lane change alert)

S4: Warning (Early Alarm State: objects very near (unsafe), fast moving objects ahead, very low response Time)

S5: Risky (Alarm State: Anytime collision state, Unexpected object crossing, front vehicle braking and no back light indication, steering out of control)

S6: Accident (collision state)

From the state diagram representation as shown in Fig. 2 the situations can be predicted and analyzed, based upon the context information provided by the middleware components.

The transition probability between states $S_{i}$ to $S_{j}$ is represented as TPM. 


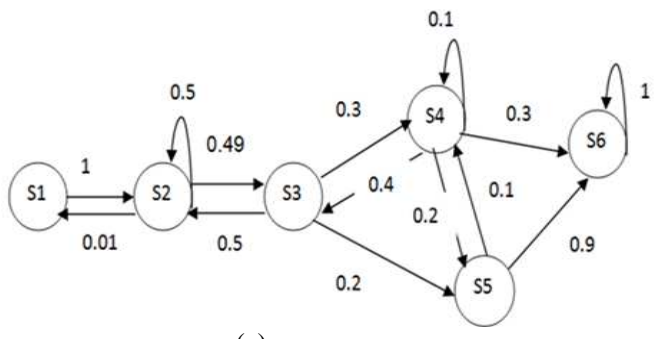

(a)
$\mathrm{TPM}=\left[\begin{array}{cccccc}0 & 1 & 0 & 0 & 0 & 0 \\ 0.01 & 0.5 & 0.49 & 0 & 0 & 0 \\ 0 & 0.5 & 0 & 0.3 & 0.2 & 0 \\ 0 & 0 & 0.4 & 0.1 & 0.2 & 0.3 \\ 0 & 0 & 0 & 0.1 & 0 & 0.9 \\ 0 & 0 & 0 & 0 & 0 & 1\end{array}\right]$

(b)

Fig. 2: (a) Markov model state diagram for context analysis (b) Transition Probability Matrix (TPM)

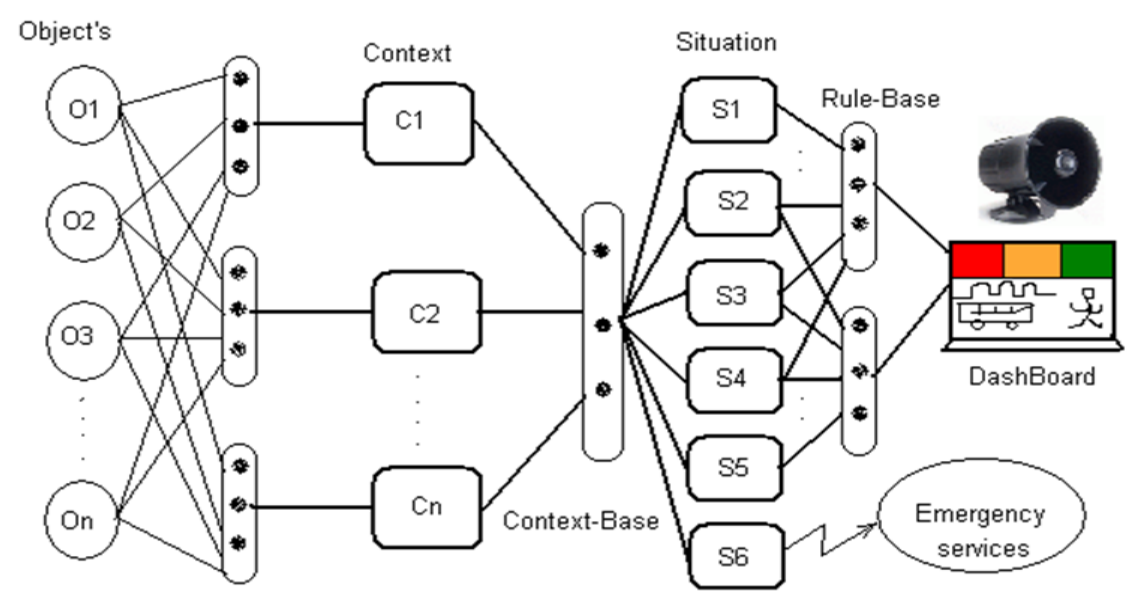

Rule-Base

Fig. 3: Context inference and sharing

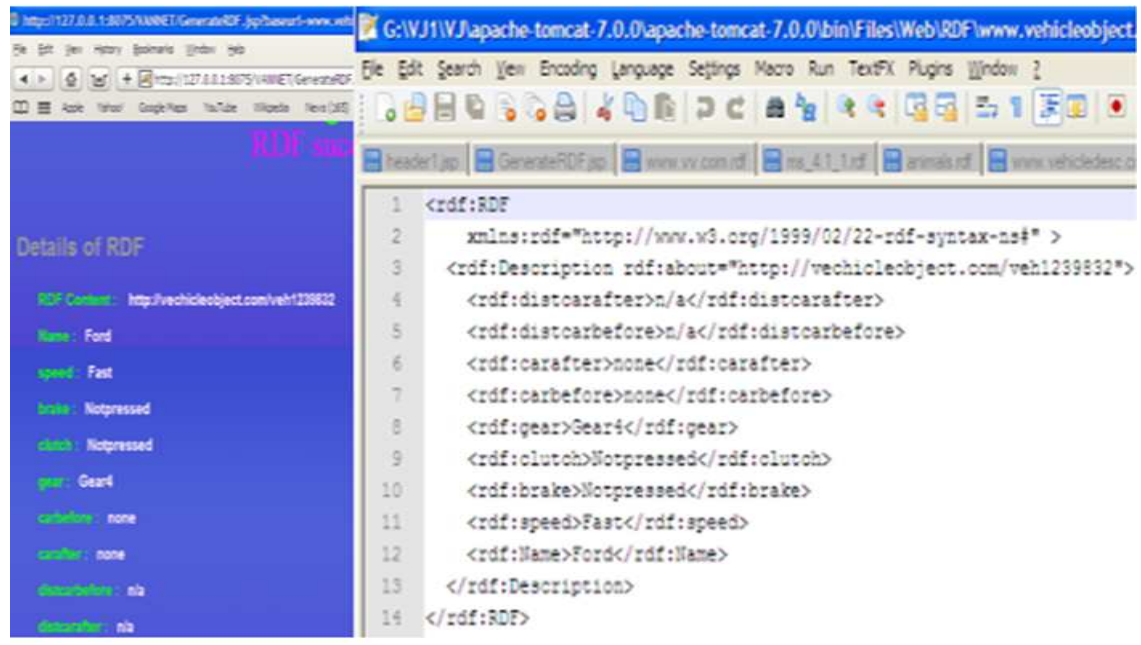

Fig. 4: Vehicle context aware information in RDF format

The initial probability values that are not constant due to the vehicle/traffic dynamics and driver response. Depending on a particular Situation the values will vary. The context-information from object is collected through three main entities such as Vehicle, Driver and
Environment. Further each entity is composed of several objects $\mathrm{O}_{1}, \mathrm{O}_{2}, \mathrm{O}_{3}, \ldots$ where some of them are highly dependent and some are not.

The context objects and its parameters considered as follows: 
O-Vehicle $=\{$ speed, $\quad$ seat-belt-position, gear-position, mp3-player, headlights, horn, indicator, braking lights, location $\}$

O-Driver $=\{$ accpedal-position, brakepedal position, Gear-position cluchpedal-position, Steering-angle $\}$

O-Environment $=$ \{ object-present, object-type, distance, Speed, angular-distance $\}$

Transition probability matrix: TPM From the State diagram, the transition probability matrix is generated to satisfy the markov process as shown in Fig.2a.

This shows the expected probability from one transition to other w.r.t. driver behavior for the current situation. Based on the current situation the alerts will be generated and notified to the driver as well as other vehicles or Road Side Units. Suppose if the Expected action is not carried over by the driver i.e., the driver reaction does not match with the threshold values defined in the policy manger, perception and decision making databases then autonomic response will be caused like controlling the vehicle through actuators and this new activity will also be added in the database for future reasoning. The main parameter to affect time critical situation is the threshold value set for the corresponding context. But the probability will vary depends on vehicle dynamics and environment conditions.

Context inference and sharing: To predict the criticality of context the observation are recorded with timestamp values and analyzed at specific time intervals. These information are stored as context database which contains heuristic and learned knowledge from the facts and inference rules as mentioned in the proposed model Fig. 1. The common way of representing aggregated context information is RDF and OWL (Hoareau and Satoh, 2009). Based upon the aggregated information the situation has been analyzed and notified accordingly.

Figure 3 shows the context inference and notification for a vehicle during critical drive away. The critical drive away scenario can occur to chances of driver in unconditional situation during drunken situations, abrupt vehicle failure, unacceptable vehicle component failure, environmental situations and roadway out of order.

The process works by observing the environmental information from context objects, predicting the state with probability distribution defined as rule-base, analyze the context information over a sampling period and also decide about a particular situation based on predicted states from context base and share the context according to the situation. The policy manager determines the chances of critical prediction of path with the existing set of calibration parameters which determines the chances of critical accident to happen and ultimately issues policy to alter situation or continue safe drive. Also updates on the last set of driving and roadway traffic parameters for future policy determination.

Figure 4 shows the RDF generation for context aware vehicle information which aids in policy management.

\section{MATERIALS AND METHODS}

CarSim Simulator (CarSim) is used for simulating the behavior of vehicles in response to driver inputs like steering, braking and acceleration.
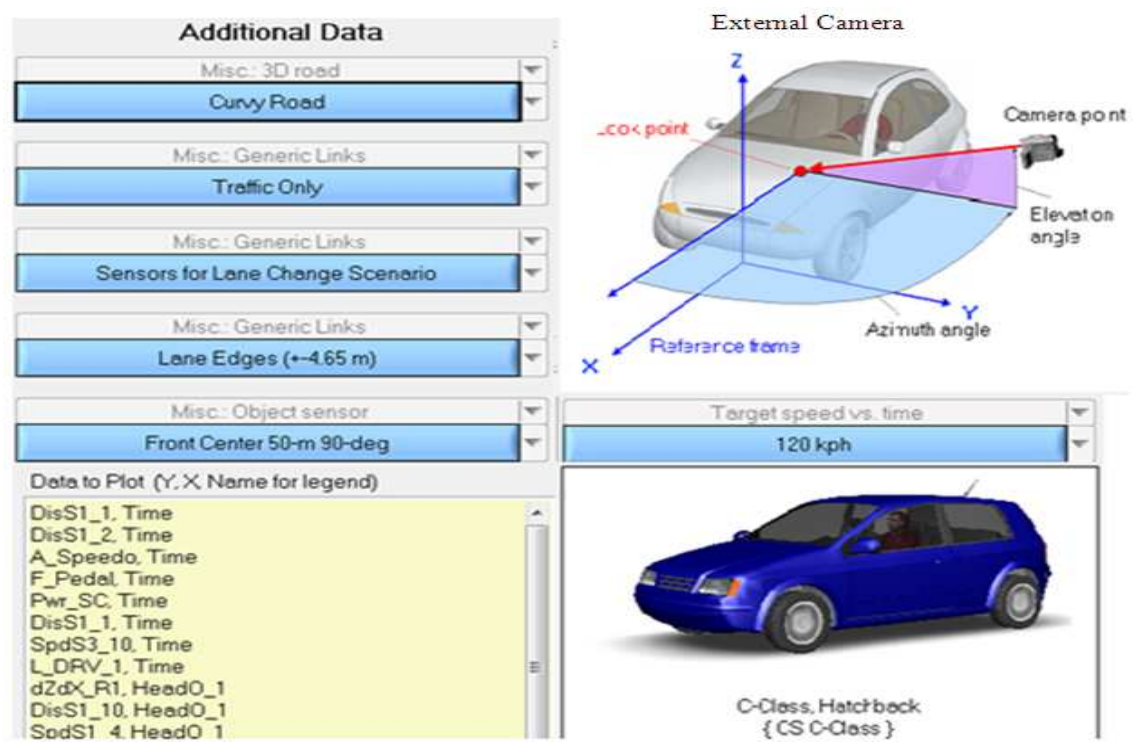

Fig.5: Carsim simulation model and test parameter 


\section{J. Computer Sci., 8 (8): 1244-1252, 2012}

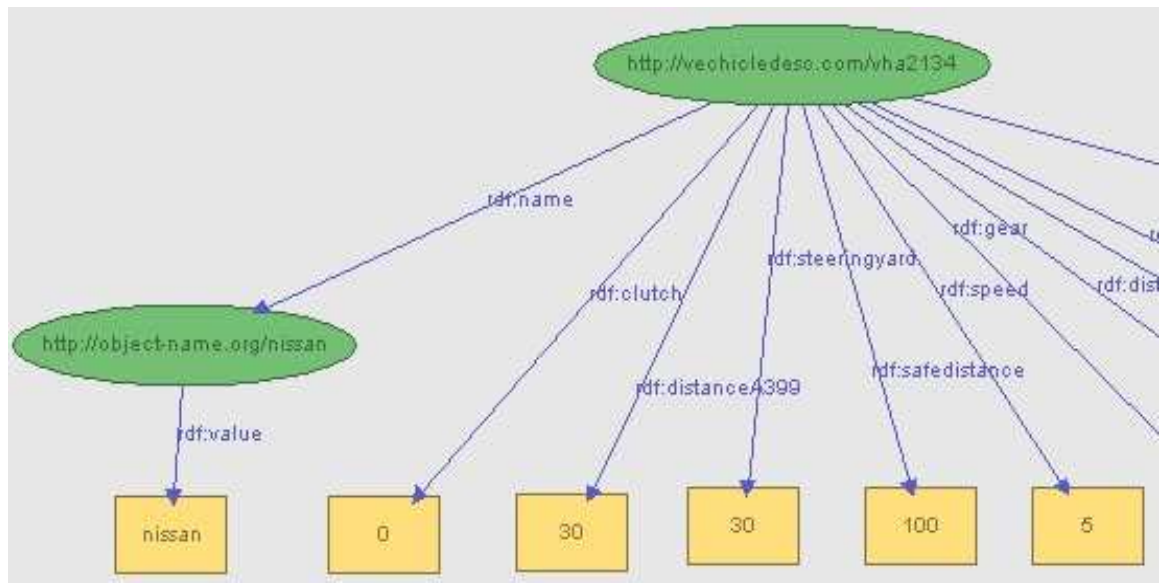

Fig.6: Owl representation of vehicle context (IsaViz)

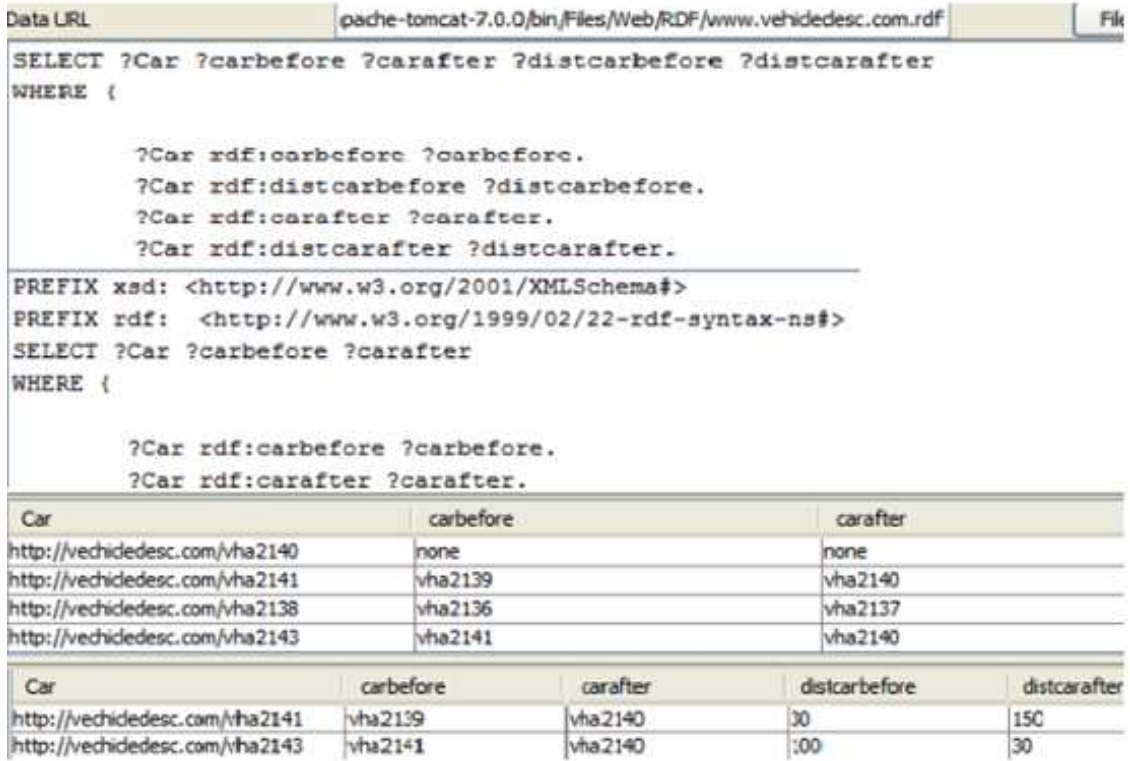

Fig. 7: Object Detection and its distance (SPARQL)

CarSim is designed to provide accurate simulation and impact on decisions much earlier in the design process. It includes various libraries for vehicle parameters, control and environmental input for simulation runs, post processing control, plot setup, animation setup and batch controls.

The simulated test information for different objects and road types are obtained from CarSim simulator (Fig. 5 ) and represented in parse file. For context prediction and analysis, the parse file information generated from CarSim has been converted in XML format to enable middleware functionalities. These files are used in Jena Platform to generate the RDF for situation analysis. The
Context information is represented in OWL format using IsaViz shown in Fig. 6.

To retrieve the criticality about the context information SPARQL queries are used. A sample query for detecting the nearby objects along with its distance is shown in Fig.7.

The context test parameters considered are:

- Brake Applied Status

- Acceleration Position

- Vehicle Length, Width

- Speed 
- Distance

- Lateral Offset

Relative Position

\section{RESULTS AND DISCUSSION}

Analysis of context prediction, inference and sharing over InVANET for improving safety in vehicle driving is carried out from predicted context for distracted driver notification to avoid accidents.

With the help of context-base, 10 types of alerts were tested for environmental, vehicle, driver and temporal context parameters with the threshold values represented in Table 1.

The different context parameters are applied to test the performance of simulation test bed on which lateral offset and longitudinal speed are discussed here to provide safety in a distracted driver situation.

Lateral offset is the horizontal distance measured from the edge of the traveled way, to the face of a roadside object or feature. This has been identified as a controlling criterion that has substantial importance in road safety Assessment, 2011 such that special attention should be given to the design decision.

The results generated for lateral offset of a vehicle in distracted state as shown Fig. 8. Without context inference and sharing mechanism the steering will be high and state of a driver becomes critical. The context about the offset of roadside object is inferred and notified to the driver in advance thereby necessary actions can be carried out by the driver to reduce the consequences. Similar results for longitudinal speed of distracted driver as shown in Fig. 9.

Table 1: Context type, expected behavior and notification

\begin{tabular}{|c|c|c|c|c|}
\hline Context type & Threshold/interface values & Observed context & Notification & Expected action \\
\hline \multirow{6}{*}{$\begin{array}{l}\text { 'Environment' } \\
\text { object detection }\end{array}$} & RADAR/ultrasonic & object ahead & Dashboard object icon & Speed in control \\
\hline & sensor interface & (can be static & with 'green' or alter & \\
\hline & $>150 \mathrm{~m}$ & or moving) & speed bumper ahead & \\
\hline & RADAR/ultrasonic & Object ahead and & Alarm (beep) dashboard & Deceleration/pre \\
\hline & $\begin{array}{l}\text { sensor interface } \\
>=50 \mathrm{~m}\end{array}$ & $\begin{array}{l}\text { safe distance (depending } \\
\text { on speed of vehicle) }\end{array}$ & object icon with 'Orange' & -safe braking \\
\hline & $\begin{array}{l}\text { RADAR/ultrasonic } \\
\text { sensor interface } \\
<10 \mathrm{~m}\end{array}$ & $\begin{array}{l}\text { Object ahead and } \\
\text { unsafe }\end{array}$ & $\begin{array}{l}\text { Alarm (beep twice) } \\
\text { dashboard object icon }\end{array}$ & $\begin{array}{l}\text { Braking/hard } \\
\text { braking/steering } \\
\text { with 'Red' }\end{array}$ \\
\hline $\begin{array}{l}\text { Environment } \\
\text { distance of any } \\
\text { moving object }\end{array}$ & $\begin{array}{l}\text { RADAR/ultrasonic } \\
\text { sensor interface } \\
<=5 \mathrm{~m}\end{array}$ & Object are very near & $\begin{array}{l}\text { Risky continuous } \\
\text { beep dashboard object } \\
\text { icone with 'Red' }\end{array}$ & $\begin{array}{l}\text { Brake and } \\
\text { maintain safe } \\
\text { distance }\end{array}$ \\
\hline $\begin{array}{l}\text { 'Vehicle' difference } \\
\text { in speed (current }\end{array}$ & $\begin{array}{l}\text { RADAR/ultrasonic } \\
\text { sensor interface } 10-30\end{array}$ & $\begin{array}{l}\text { Slow moving } \\
\text { vehicle ahead }\end{array}$ & $\begin{array}{l}\text { Alert (beep) dashboard } \\
\text { object icon with 'Orange' }\end{array}$ & $\begin{array}{l}\text { Deceleration/pre } \\
\text {-safe braking }\end{array}$ \\
\hline $\begin{array}{l}\text { vehicle and } \\
\text { front vehicle) }\end{array}$ & $\begin{array}{l}\mathrm{kmph} \text { and current speed } \\
\text { is }<=40 \mathrm{kmph}\end{array}$ & & & \\
\hline Temporal & $\begin{array}{l}\text { Time interface } \\
\text { time and day }\end{array}$ & $\begin{array}{l}\text { Early morning (2-5) } \\
\text { morning (5-12)Mon- } \\
\text { Fri/noon (12-4) Sat, } \\
\text { Sun evening (4-6) night } \\
\text { (6-11) Midnight (11-2) }\end{array}$ & $\begin{array}{l}\text { Traffic view/current } \\
\text { status/prediction } \\
\text { based on database }\end{array}$ & $\begin{array}{l}\text { Steady in driving } \\
\text { all the time, more } \\
\text { cautious at midnight/ } \\
\text { early morning }\end{array}$ \\
\hline $\begin{array}{l}\text { 'Environment' } \\
\text { location of vehicle }\end{array}$ & $\begin{array}{l}\text { GPS device interface } \\
\text { (longitude, latitude) }\end{array}$ & $\begin{array}{l}\text { Present location of } \\
\text { the vehicle }\end{array}$ & Route guidance & following the route \\
\hline 'Vehicle /driver' & Crash sensor interface vehicle & Collision vehicle problems & Autonomic message & Rescue/emergency \\
\hline Accident & stopped due to collision & driver health condition & $\begin{array}{l}\text { call for emergency } \\
\text { service (SOAP-WS) }\end{array}$ & service \\
\hline
\end{tabular}

Lateral offset from design path-m

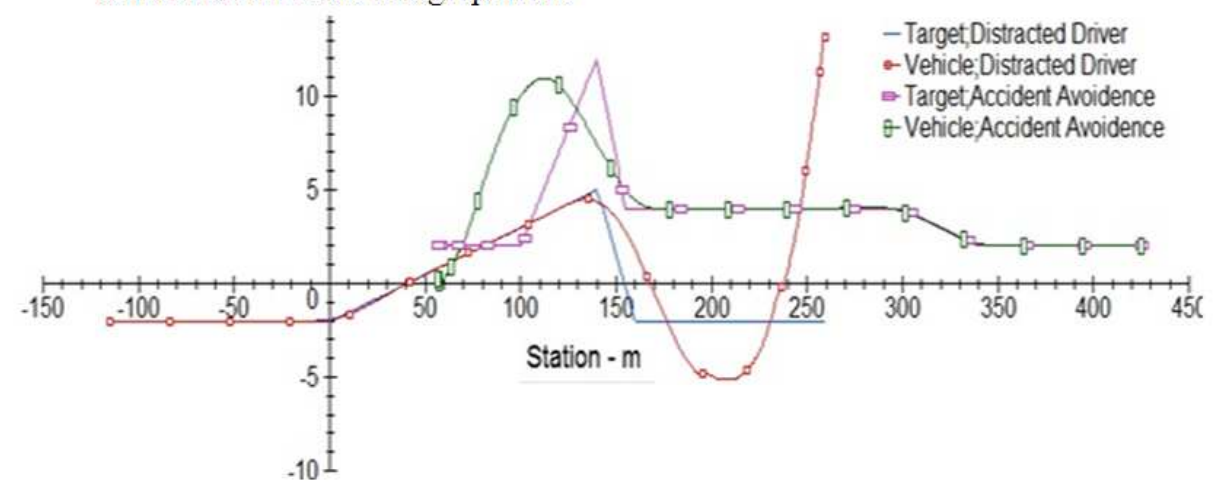

Fig.8: Lateral offset of vehicle in distracted state 


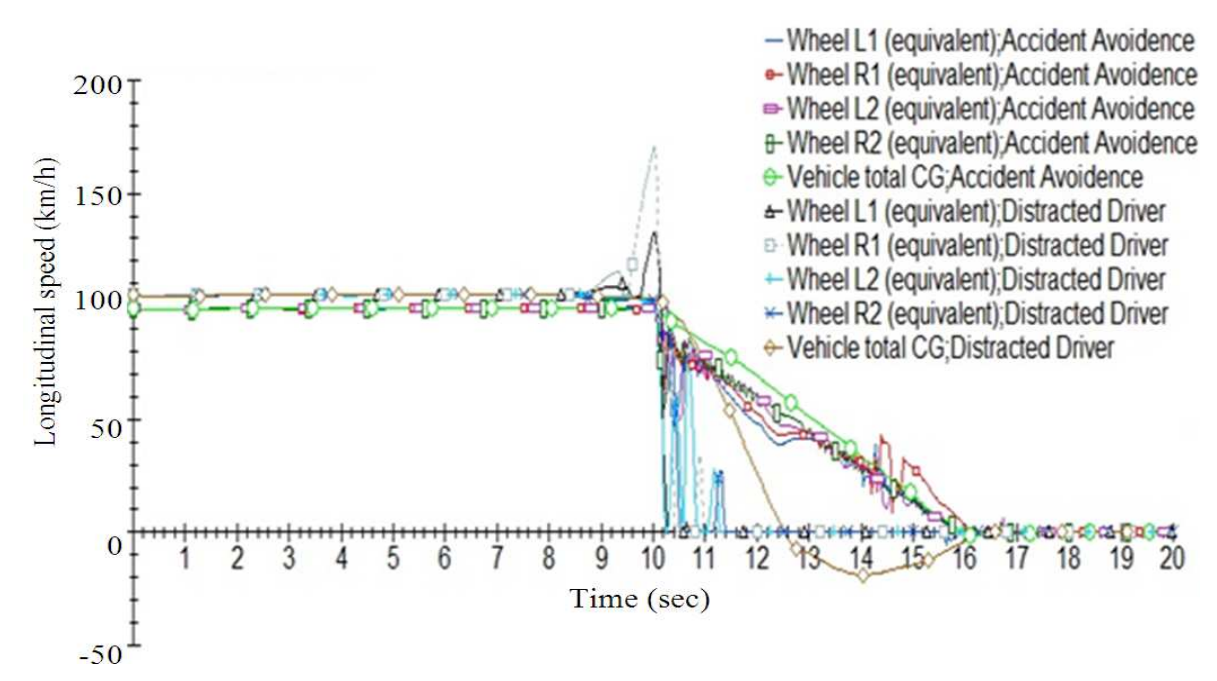

Fig.9: Distracted driver and accident avoidance

\section{CONCLUSION}

InVANET can be identified as a promising system for preventing various types of collisions, ranging from driver warning systems to vehicle control devices.

Through context modeling for InVANET with different interpretation mechanism and simulation of middleware system shows that identifying critical situations and issue alerts can be done for more than $70 \%$ of roadway safety scenarios. Even though it helps in minimizing accidents and improving safety, basic QoS issues and security mechanisms are to be improved.

The application of context prediction and inference over InVANET raises several interesting issues in regard to context sharing. Context about environment may not be accurate sometimes due to large number of message flow between vehicles and carriers. The time sensitive information should be prioritized and validated in dynamic traffic environments. Another issue to consider is security in context message transactions. If a vehicle send a message which is not trusted and vehiclecarriers are forwarding same message to neighborhood there will be chances on spreading wrong information on the network. Hence QoS issues and security are to be considered as future research.

\section{ACKNOWLEDGMENT}

Acknowledgments are due to www.vit.ac.in for encouraging us to carry out the research, Carsim (www.carsim.com) and Net Research Lab forum (www.netresearchlabs.net) members for guiding and helping in setting up test-bed and conducting research.

\section{License:}

- Atmel Mega32 MCU card is trade mark of Atmel Corp (www.atmel.com)

- CarSim Simulation, www.carsim.com

\section{REFERENCES}

Andreas, F., 2004. FleetNet: Bringing car-to-car communication into the real world. Proceedings of 11th World Congress on ITS, (WCITS' 04), Nogoya, Japan.

Arunkumar, T. and S.N. Sivanandam, 2007. Location identification and vehicular tracking for vehicular ad-hoc wireless networks.

Babich, F. and G. Lombardi, 2000. A markov model for the mobile propagation channel. IEEE Trans. Vehicular Technol., 49: 63-73. DOI: $10.1109 / 25.820699$

Benoit, M., 2002. IVHW inter-vehicle hazard warning system. Federal Ministry of Education and Research.

Festag, A., G. Noecker, M. Strassberger, A. Lubke and B. Bochow et al., 2008. 'NoW-network on wheels': Project objectives, technology and achievements. Proceedings of 6th Internatioinal Workshop on Intellligent Transportation, (IT' 08), Hamburg, Germany, pp: 211-216.

Gerhard, N., 2004. Goal, Scenarios and Requirements. WILLWARN-Wireless Local Danger Warning.

Gura, N., A. Held and J. Kaiser, 2001. Proactive Services in a distributed traffic telematics application. Porceedings of the GI Jahrestagung, (GIJ' 01), Odysci, pp: 585-592. 
Hoareau, C. and I. Satoh, 2009. Modeling and processing information for context-aware computing: A survey. New Generation Comput., 27: 177-196. DOI: 10.1007/s00354-009-0060-5

Nagappan, G. and C. Chellappan, 2012. Intelligent model for traffic safety applications. J. Comput. Sci., 8: 358-365. DOI: 10.3844/jcssp.2012.358.365

Reichardt, D., M. Miglietta, L. Moretti, P. Morsink and W. Schulz, 2002. CarTALK 2000: Safe and comfortable driving based upon inter-vehicle-communication. Proceedings of the Intelligent Vehicle Symposium, Jun. 17-21, IEEE Xplore Press, pp: 2: 545- 550. DOI: 10.1109/IVS.2002.1188007

Sadayuki, T., 2011. Automated driving systems: Common ground of automobiles and robots. J. Humanoid Robotics, 8: 1-12. DOI: 10.1142/S0219843611002319

Saravanan, K., A. Thangavelu and K. Rameshbabu, 2009. A Middleware Architectural framework for Vehicular Safety over VANET (InVANET). Proceedings of the ACM DL and IEEE First International Conference on Networks and Communications, NETCOM, Dec. 27-29. IEEE Xplore Press, India, pp: 277-282. DOI: 10.1109/NetCoM.2009.57

Shankar, P., T. Nadeem, J. Rosca and L. Iftode, 2008. CARS: Context-Aware Rate Selection for vehicular networks. Proceedings of the IEEE International Conference on Network Protocols ICNP, IEEE Xplore Press, New Jeresy, pp: 1-12. DOI: 10.1109/ICNP.2008.4697019
Shengpu, L. and C. Liu, 2011. A context-aware reflective middleware framework for distributed real-time and embedded systems. J. Syst. Software, 84: 205-218. DOI: 10.1016/j.jss.2010.09.049

Shladover, S.E., 2007. PATH at 20-history and major milestones. IEEE Trans. Intell. Transportation Syst., 8: 584-592. DOI: 10.1109/TITS.2007.903052

Wassim, G., N. John, D. Smith and M. Yanagisawa, 2007. Pre-Crash Scenario Typology for Crash Avoidance Res. National Highway Traffic Safety Administration.

WHO, 2009. World health organization global status report on road safety. World Heath Organization.

Williams, M., 1988. PROMETHEUS-The European research programme for optimising the road transport system in Europe. IEE Colloquium Driver Inform.

Wu, Z., Q. Wu, H. Cheng, G. Pan and M. Zhao et al., 2007. ScudWare: A semantic and adaptive middleware platform for smart vehicle space. IEEE Trans. Intell. Transportation Syst., 8: 121-132. DOI: 10.1109/TITS.2006.890080 